\title{
Extremely large magnetoresistance from electron-hole compensation in the nodal-loop semimetal $\mathrm{ZrP}_{2}$
}

\author{
J. Bannies $\odot,{ }^{1,2, *}$ E. Razzoli, ${ }^{2,3}$ M. Michiardi, ${ }^{2,3,1}$ H.-H. Kung $\odot,{ }^{2,3,1}$ I. S. Elfimov, ${ }^{2,3}$ M. Yao, ${ }^{1}$ A. Fedorov, ${ }^{4,5}$ J. Fink $\odot,{ }^{1,4,6}$ \\ C. Jozwiak @,$^{7}$ A. Bostwick, ${ }^{7}$ E. Rotenberg, ${ }^{7}$ A. Damascelli $\odot{ }^{2,3}$ and C. Felser ${ }^{1, \dagger}$ \\ ${ }^{1}$ Max Planck Institute for Chemical Physics of Solids, 01187 Dresden, Germany \\ ${ }^{2}$ Quantum Matter Institute, University of British Columbia, Vancouver, British Columbia, V6T 1Z4, Canada \\ ${ }^{3}$ Department of Physics \& Astronomy, University of British Columbia, Vancouver, British Columbia, V6T 1Z1, Canada \\ ${ }^{4}$ Leibniz Institute for Solid State and Materials Research Dresden, 01069 Dresden, Germany \\ ${ }^{5}$ Helmholtz-Zentrum Berlin für Materialien und Energie, 12489 Berlin, Germany \\ ${ }^{6}$ Institut für Festkörperphysik, Technische Universität Dresden, 01062 Dresden, Germany \\ ${ }^{7}$ Advanced Light Source (ALS), Berkeley, California 94720, USA
}

(Received 20 October 2020; accepted 22 March 2021; published 22 April 2021)

\begin{abstract}
Several early transition metal dipnictides (TMDPs) have been found to host topological semimetal states and exhibit large magnetoresistance (MR). In this paper, we use angle-resolved photoemission spectroscopy (ARPES) and magnetotransport to study the electronic properties of a $\mathrm{TMDP} \mathrm{ZrP}_{2}$. We find that $\mathrm{ZrP}_{2}$ exhibits an extremely large and unsaturated MR of up to $40000 \%$ at $2 \mathrm{~K}$, which originates from an almost perfect electron-hole (e-h) compensation. Our band structure calculations further show that $\mathrm{ZrP}_{2}$ hosts a topological nodal loop in proximity to the Fermi level. Based on the ARPES measurements, we confirm the results of our calculations and determine the surface band structure. This paper establishes $\mathrm{ZrP}_{2}$ as a platform to investigate near-perfect e-h compensation and its interplay with topological band structures.
\end{abstract}

DOI: 10.1103/PhysRevB.103.155144

\section{INTRODUCTION}

Topological semimetals have attracted considerable scientific interest owing to their unique electronic structure and unconventional transport properties, such as extremely large magnetoresistance (XMR) [1-6] and planar Hall effect [7,8], as well as their deep connection with high-energy particle physics [9]. These unusual properties not only pave the way for future electronic and spintronic devices, but also provide a test bed for our current models of transport theories [10]. The characteristic dispersion feature of most topological semimetals is a linear intersection of quasiparticle bands near the Fermi energy. In the most well-studied examples, i.e., Dirac $[11,12]$ and Weyl semimetals $[1,13,14]$, the energy bands intersect each other on a single point in $k$-space with fourfold and twofold degeneracies, respectively. Nodal line semimetals, on the other hand, exhibit band crossings that extend on a one-dimensional path in $k$-space [15], which can be an open line, a closed loop, or even loops with complex connectivity [16-19]. Examples of nodal line semimetals have been found in $\mathrm{ZrSiS}$-type materials [20-23] and early transition metal

*jbannies@chem.ubc.ca

†claudia.felser@cpfs.mpg.de

Published by the American Physical Society under the terms of the Creative Commons Attribution 4.0 International license. Further distribution of this work must maintain attribution to the author(s) and the published article's title, journal citation, and DOI. Open access publication funded by the Max Planck Society. dipnictides (TMDPs) $M X_{2}(M=\mathrm{Nb}, \mathrm{Ta}, \mathrm{Mo}, \mathrm{W} ; X=\mathrm{P}, \mathrm{As}$, $\mathrm{Sb}$ ) in the absence of spin-orbit coupling (SOC) [24-27].

In early TMDPs, nontrivial topology is often accompanied by unsaturated XMR $[1,3,28-31]$. Elements highlighted in Fig. 1(a) all exhibit XMR in TMDPs. While XMR has been found for TMDPs made of group V and VI metals, as highlighted in Fig. 1(a), no group IV TMDPs have been studied. Here, we investigate $\mathrm{ZrP}_{2}$ as a case study that demonstrates the ubiquity of these properties in early TMDPs. We propose that two factors are key for understanding the relation between topology and XMR in these compounds: nonsymmorphic crystal symmetry and partial charge transfer in the bonding.

Most of known early TMDPs crystallize either in an $\mathrm{OsGe}_{2}$ type (space group $C 2 / m$, No. 12) or $\mathrm{MoP}_{2}$ type structure (space group $\mathrm{Cmc}_{1}$, No. 36), as shown in Figs. 1(b) and 1(c) [32]. In the $\mathrm{MoP}_{2}$ structure, the four transition metal (TM) sites are related by three glide planes $(b, c$, and $n)$, whereas in the $\mathrm{OsGe}_{2}$ structure, two pairs of TM sites are related by one glide plane $(a)$. In both cases, the conventional unit cell therefore contains four and the primitive unit cell two formula units. The nonsymmorphic symmetry thus indirectly guarantees an even number of valence electrons, regardless of the atomic number of the TM. Moreover, the effective doublings of the unit cell cause band back-folding, facilitating the occurrence of band crossings such as nodal lines.

Considering that the $d$ electrons of early TMs are relatively weakly bound and easily transferable to the pnictogen [33], we would expect to see fully filled valence bands and empty conduction bands, consistent with a band insulator with no charge carriers. However, early TMDPs consistently show metallic conductivity in experiments $[32,33]$. This inconsistency is resolved by assuming that the charge transfer 
(a)

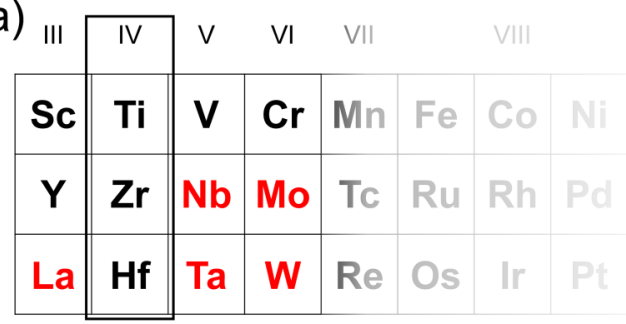

(d)

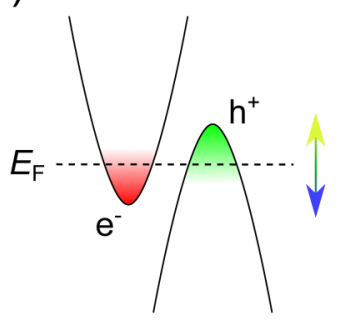

(b)

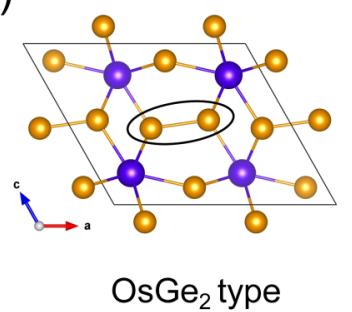

(e)
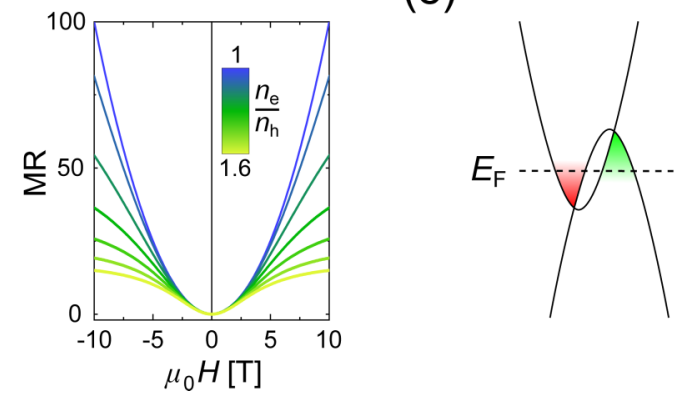

(c)

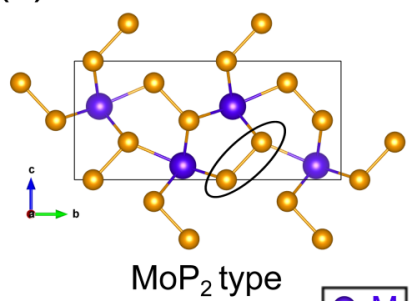

(f)

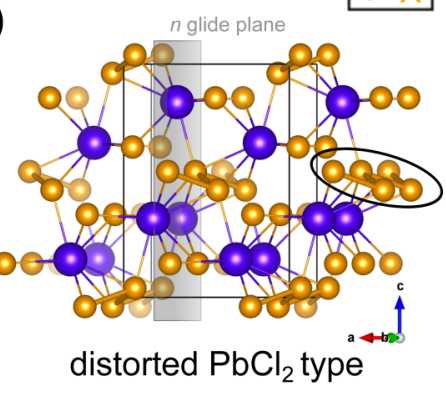

FIG. 1. Occurrence of extremely large magnetoresistance (XMR) in early transition metal dipnictides (TMDPs). (a) Excerpt of the periodic table. Elements for which at least one TMDP was found to show XMR are marked in red. (b) and (c) Common crystal structure types of early TMDPs: OsGe 2 type and $\mathrm{MoP}_{2}$ type, both containing glide planes and two formula units per primitive unit cell. Covalent bonding motifs of anions are encircled. (d) Schematic of a trivial semiclassical two-band model (left). Simulated magnetoresistance (MR) for different carrier density ratios (right). Mobilities: $\mu_{h}=\mu_{e}=1 \mathrm{~m}^{2} / \mathrm{Vs}$. (e) Schematic of a nontrivial compensated two-band system. (f) Distorted PbCl $\mathrm{P}_{2}$-type crystal structure as found in $\mathrm{ZrP}_{2}$. One of the nonsymmorphic symmetry elements, the $n$ glide plane, is shown.

is incomplete, resulting in semimetallic Fermi surfaces. The electronlike and holelike charge carriers' densities are hence naturally close to compensated, leading to an unsaturated XMR within the semiclassical approximation [Fig. 1(d)] $[34,35]$. We note that the above statements should generally hold true for all early TMDPs. However, to the best of our knowledge, no group IV TMDP has ever been reported to display nonsaturating XMR, leaving an open question to the generality of the above simple arguments based on nonsymmorphic symmetry and incomplete charge transfer.

Here, we demonstrate that $\mathrm{ZrP}_{2}$ is a group IV dipnictide that exhibits nonsaturating XMR. The electron and hole carrier densities determined from magnetotransport measurements are almost perfectly compensated, which quantitatively agrees with the observed XMR [34]. The electron-hole (e-h) compensation is further confirmed by density functional theory (DFT) calculations, which also reveal the existence of a nodal loop below the Fermi level in the $k_{x}=0$ plane. Our band structure calculations are verified by angle-resolved photoemission spectroscopy (ARPES).

\section{METHODS}

Crystals of $\mathrm{ZrP}_{2}$ were grown by a two-step process. Stoichiometric mixtures of zirconium (Alfa Aesar, $99.7 \%$, foil) and red phosphorus (Alfa Aesar, $99.995 \%$, pieces) with an addition of iodine were prereacted at $800^{\circ} \mathrm{C}$ for $7 \mathrm{~d}$. Subsequently, crystals were grown by horizontal chemical vapor transport using a temperature gradient from $760{ }^{\circ} \mathrm{C}$ (source) to $810{ }^{\circ} \mathrm{C}$ (sink) with $7.5 \mathrm{mg} / \mathrm{mL}$ iodine as a transport agent. After $10 \mathrm{~d}$, needlelike crystals of typical dimensions
$0.3 \times 0.3 \times 5 \mathrm{~mm}^{3}$ were obtained in the sink. Details of the crystal characterization are given in the Supplemental Material [36]. The electrical transport and Hall measurements were performed with a conventional five-probe geometry on a 9 T PPMS (Quantum Design). The Hall data were antisymmetrized to remove contributions from the transverse magnetoresistance (MR).

ARPES measurements were performed at the microARPES endstation of the MAESTRO beamline 7.0.2 at Advanced Light Source (ALS) and the $1^{2}$ endstation of beamline UE112 PGM at BESSY. Samples were cleaved and measured at $15 \mathrm{~K}(20 \mathrm{~K})$ and pressures lower than $4 \times 10^{-11}$ torr $\left(1 \times 10^{-10}\right.$ torr) at ALS (BESSY). Energy and angular resolution at both endstations are better than $20 \mathrm{meV}$ and $0.2^{\circ}$, respectively.

Electronic structure calculations were performed within the framework of DFT as implemented in the package Wien2k [37]. The generalized gradient approximation with the Perdew-Burke-Ernzerhof parametrization [38] was used. Due to the semimetallicity of $\mathrm{ZrP}_{2}$, calculations were also performed with the modified Becke-Johnson functional [39]. The basis set size was set to $\mathrm{R}_{m t} \mathrm{~K}_{\max }=7$. For bulk calculations, the irreducible Brillouin zone (BZ) was sampled with $1440 \mathrm{k}$ points. Surface band structures were obtained from slab calculations. Slabs were constructed by stacking five unit cells along $c$ separated by $20 \AA$ vacuum. The BZ was sampled with a $16 \times 30 \times 3 \mathrm{k}$ mesh.

\section{RESULTS AND DISCUSSION}

$\mathrm{ZrP}_{2}$ crystallizes in a distorted $\mathrm{PbCl}_{2}$ structure [Fig. 1(f)] belonging to the nonsymmorphic space group Pnma (No. 
(a)

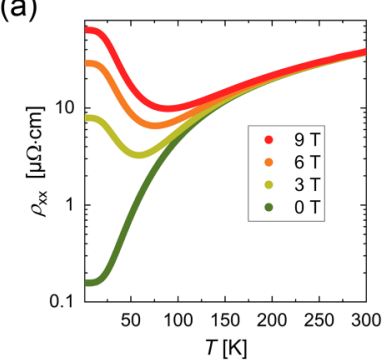

(c)

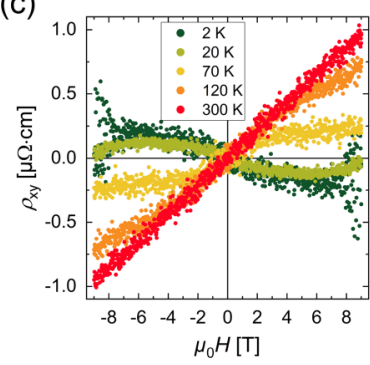

(b)

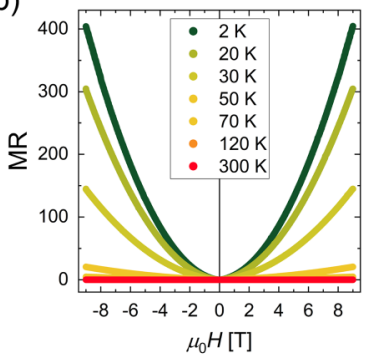

(d)

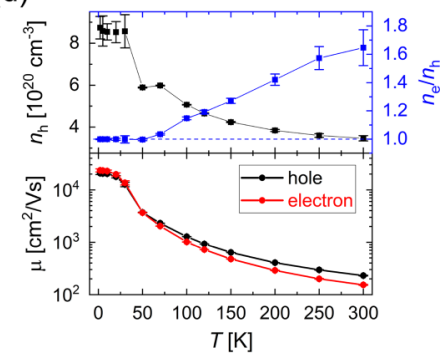

FIG. 2. Electrical transport behavior of $\mathrm{ZrP}_{2}$ evidencing a semimetallic state. (a) Temperature dependence of $\rho_{x x}$ in different magnetic fields with I $\| b$. (b) and (c) Magnetic field dependence of magnetoresistance (MR) and $\rho_{x y}$ at selected temperatures. (d) Charge carrier densities and mobilities obtained by simultaneously fitting MR and $\rho_{x y}$ to the two-band model [34]. Note that the large uncertainties of $n_{h}$ compared with $n_{e} / n_{h}$ below $50 \mathrm{~K}$ originate from the fits. Depending on the initial values for the fit, values of $n_{h}$ converged only to the window given by the uncertainties. In contrast, independent of the final value of $n_{h}, n_{e} / n_{h}$ is always $\sim 1$.

62, $D_{2 \mathrm{~h}}^{16}$ ) [40]. Half of the $\mathrm{P}$ atoms form chains with short P-P bonds $(2.34 \AA)$ along the $b$ axis, indicating a covalent bonding character between negatively charged $\mathrm{P}$ atoms [Fig. 1(f)]. These chains provide a preferred direction of growth. Indeed, crystals obtained from chemical vapor transport exhibit needlelike shape with the crystallographic $b$ axis oriented along the needle axis. The lattice parameters $a=6.4933(2) \AA, b=3.5120(1) \AA$, and $c=8.7431(3) \AA$, determined from powder X-ray diffraction (XRD), agree with previously reported values [40].

The resistivity measurements reveal metallic behavior with a low residual resistivity of $0.18 \mu \Omega \mathrm{cm}$ at $2 \mathrm{~K}$ [Fig. 2(a)]. The high residual resistivity ratio of 238 is an indication of the good quality of the crystals. Notably, a field-induced metal-toinsulatorlike transition is observed above $3 \mathrm{~T}$ and below $50 \mathrm{~K}$ [Fig. 2(a)], similar to other topological semimetals [1,28-30]. As shown in Fig. 2(b), the MR, which is defined as $\mathrm{MR}=$ $\rho(B) / \rho(B=0)-1$, remains unsaturated in fields up to $9 \mathrm{~T}$ and reaches $40000 \%$ at $2 \mathrm{~K}$ and $9 \mathrm{~T}$. The field dependence of the MR is close to parabolic and can be fitted to a power law $\mathrm{MR}=a+b B^{c}$ with $c=1.92$.

To investigate the origin of the large and unsaturated MR in detail, we performed Hall measurements where the resistivity $\rho_{x y}(B)$ is perpendicular to both the field and electric current. The $\rho_{x y}(B)$ curves [Fig. 2(c)] feature a distinct nonlinearity that can be fitted with a semiclassical two-band model [34]. We note that the noise level of these curves is inherent to $\mathrm{ZrP}_{2}$ due to the narrow width of the crystals. By simultaneously fitting MR and Hall resistivity to the two-band model, we

obtained carrier densities $n_{e, h}$ and mobilities $\mu_{e, h}$, as shown in Fig. 2(d). At room temperature, electrons are the dominant charge carriers. Upon cooling, the ratio $n_{e} / n_{h}$ decreases, and the charge carriers become almost completely compensated below $50 \mathrm{~K}$, with $n_{e} / n_{h}=0.999(2)$ at $2 \mathrm{~K}$. The mobilities of electrons and holes also show similar values for all temperatures and reach high values up to $2.3 \times 10^{4} \mathrm{~cm}^{2} / \mathrm{Vs}$ at $2 \mathrm{~K}$, which further confirms the good crystal quality. Together, our magnetotransport measurements strongly suggest that e-h compensation is the origin for the unsaturated XMR in the semimetal $\mathrm{ZrP}_{2}$.

In support for these findings, we performed DFT calculations, and the resulting band structure is presented in Fig. 3(a). $\mathrm{ZrP}_{2}$ shows semimetallicity with both electron and holelike bands crossing the Fermi level. The corresponding Fermi surface is formed by two holelike bands, $\alpha$ and $\beta$ sheets in Figs. 3(b) and 3(c), and one electronlike band $\gamma$, see Fig. 3(c). However, the volume of the $\alpha$ hole pocket is very small compared with the other pockets and can be neglected without losing soundness of the model. The description by an effective two-band model remains valid and in accordance with transport experiments. The calculated ratio of the carrier densities $n_{e} / n_{h}=1.02$, demonstrating almost perfect e-h compensation in line with the electrical transport experiments.

A close look to the low-energy band structure reveals a nodal loop in the $k_{x}=0$ plane, with energy slightly below the Fermi level, as shown in Fig. 3(e). The stability of the nodal loop against opening a gap is guaranteed by the $n$ glide plane [Fig. 1(f)], i.e., the bands have eigenvalues of opposite signs with respect to the glide reflection symmetry, as indicated in Fig 3(d). Upon inclusion of SOC, the nodal loop opens a small gap, which varies between 7 and $20 \mathrm{meV}$.

To get a conclusive picture on the electronic structure of $\mathrm{ZrP}_{2}$, we employed ARPES as a tool to directly map the band structure. The experimental bulk Fermi surface shown in Fig. 4(a) was measured with $40 \mathrm{eV}$ photons at BESSY. This photon energy was chosen to correspond to $k_{z}=0$, as determined by photon-energy dependent measurements (see Supplemental Material [36]). The data confirm the presence of both the $\beta$ hole and the $\gamma$ electron pockets, in good agreement with theory and transport experiments. The $\alpha$ hole pocket is too small to be identified, given the resolution of our ARPES data.

We further confirm the agreement between theory and ARPES by investigating the high-symmetry paths $\bar{Y}-\bar{\Gamma}-\bar{Y}$ and $\overline{\mathrm{M}}-\overline{\mathrm{X}}-\overline{\mathrm{M}}$ in a large binding energy window, as illustrated in Figs. 4(b) and 4(c). At the low photon energy used in our experiment, $k_{z}$ is ill defined as a consequence of the short mean free path of the photoemitted electron and the relatively small size of the Brillouin zone along $k_{z}$ [41]. We find that the uncertainty of $k_{z}$ is $\sim 0.6 \pi / c$ (see Supplemental Material [36] for details). Therefore, we compare the spectra with the projected bulk band structure within the $k_{z} \in[0,0.6 \pi / c]$ range and find good agreement in the entire binding energy range. We note that the band crossing point of the nodal loop along $\bar{Y}-\bar{\Gamma}-\bar{Y}$ could not be identified owing to two possibly concomitant reasons: (i) at any measured photon energy, only one of the two crossing bands is seen due to unfavorable photoemission cross-section [42] and (ii) broadening from surface disorder and $k_{z}$ integration smears out the band crossing. 
(a)

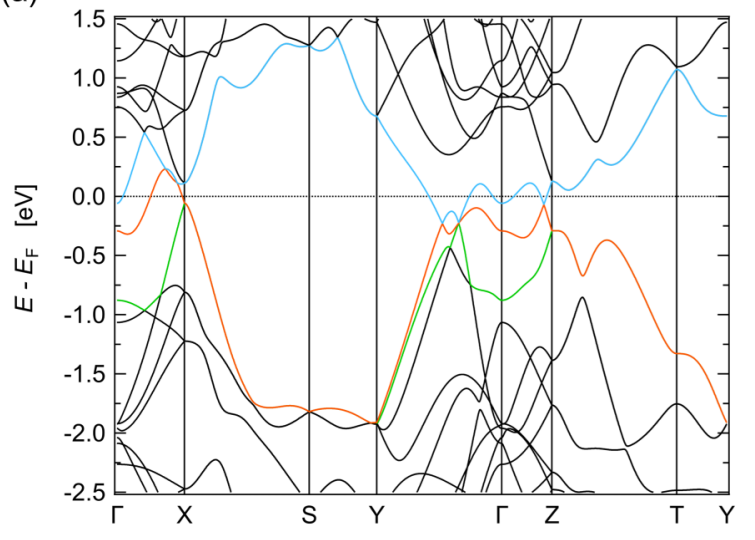

(b)

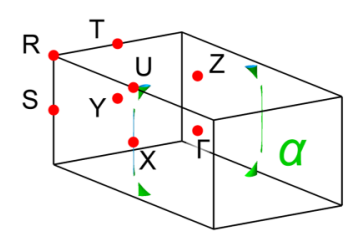

(c)

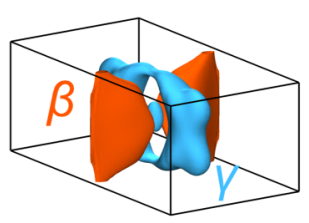

(d)

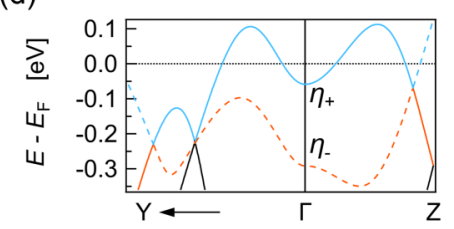

(e)

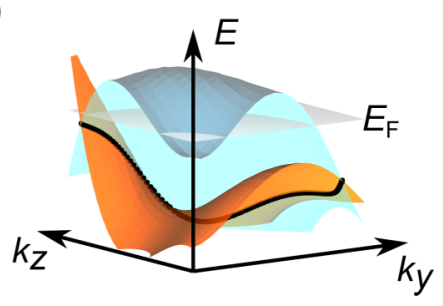

FIG. 3. Electronic structure of $\mathrm{ZrP}_{2}$ from density functional theory calculations without spin-orbit coupling. (a) Band structure with the three bands crossing $E_{F}$ colored. (b) and (c) Three-dimensional (3D) Fermi surface of $\mathrm{ZrP}_{2}$ exhibiting two hole pockets $(\alpha$ and $\beta$ ) and an electron pocket $(\gamma)$. Color coding according to panel (a). (d) Close-up of the low-energy band structure in the $k_{x}=0$ plane. Eigenvalues $\eta_{ \pm}$ with respect to the $n$ glide plane are indicated (closed line $\eta_{+}$, dashed $\eta_{-}$). (e) 3D visualization $E\left(k_{y}, k_{z}\right)$ of the $\beta$ and $\gamma$ bands forming the nodal loop (black line) in the $k_{x}=0$ plane. Only one quadrant of the $k_{x}=0$ plane is shown.

(a)

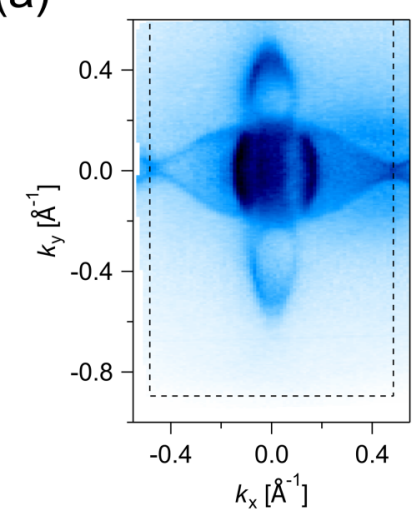

(d)

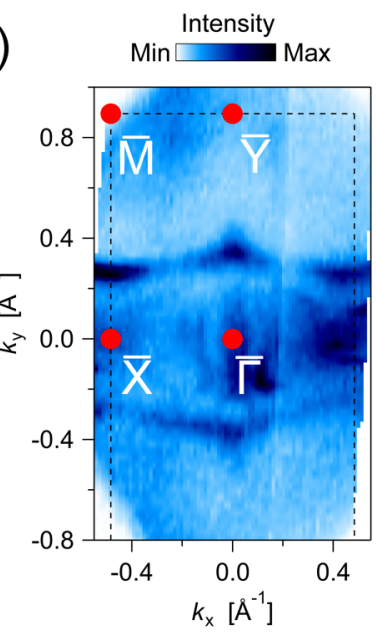

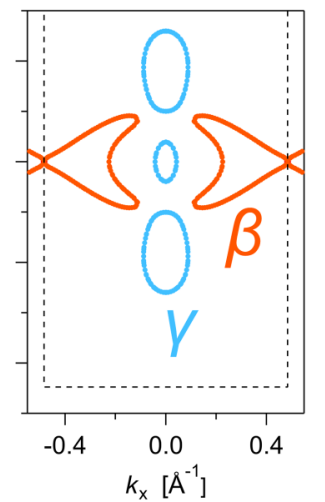

Surface contr.

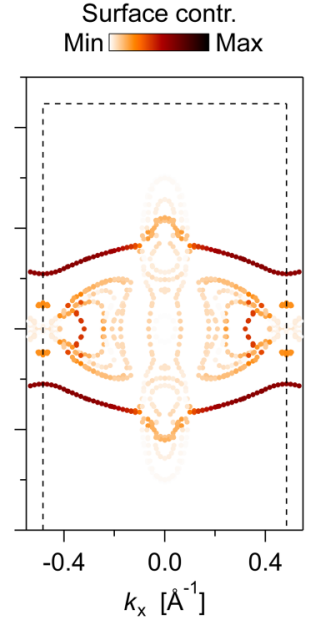

(b)

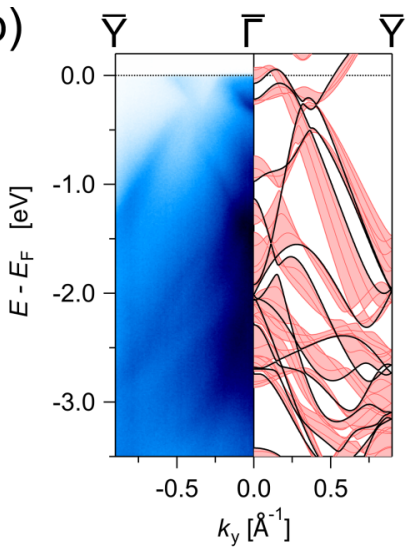

(e)

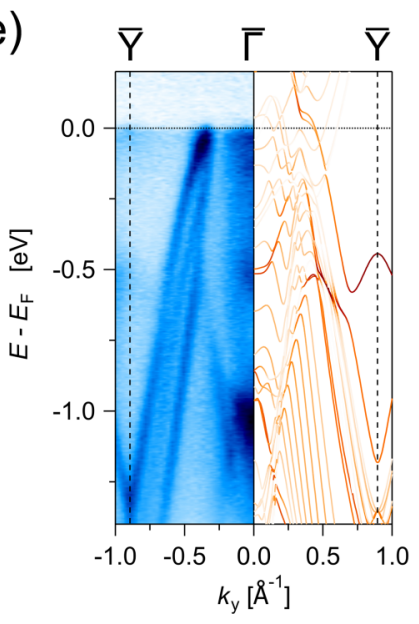

(c)

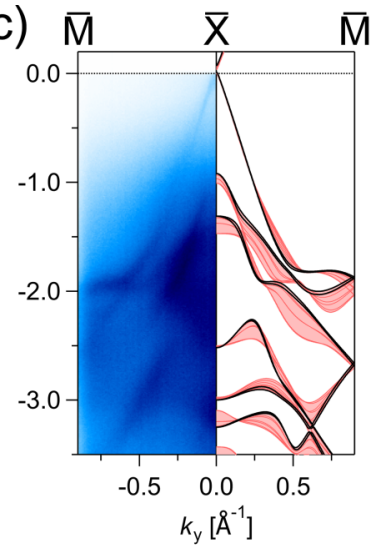

(f)

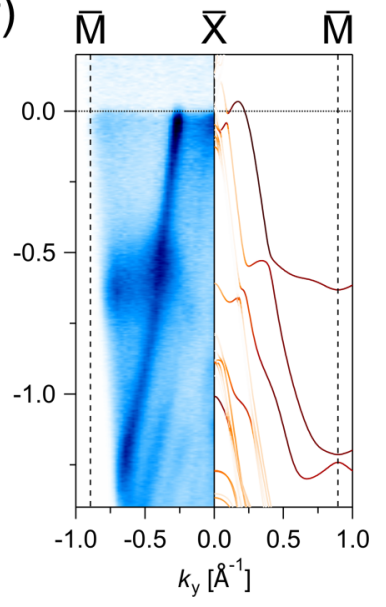

FIG. 4. Bulk and surface band structure of $\mathrm{ZrP}_{2}$ measured by angle-resolved photoemission spectroscopy (ARPES; both $h v=40 \mathrm{eV}$, linear horizontal polarization) and comparison with density functional theory calculations. (a) Bulk Fermi surface from ARPES (left) and calculations (right) both at $k_{z}=0$. (b) and (c) Cuts along $\bar{Y}-\bar{\Gamma}-\bar{Y}$ and $\bar{M}-\bar{X}-\bar{M}$ showing bulk bands, respectively. Calculations show the projected band structures for $k_{z} \in[0,0.6 \pi / c]$, where bands with $k_{z}=0$ are highlighted in black. (d)-(f) Data acquired at Advanced Light Source, where the small spot size allowed us to focus on an area with a single termination, emphasizing the surface band structures [36]. Note that the calculated surface band structure was shifted by $50 \mathrm{meV}$ with respect to the theoretical Fermi level to match the experimental dispersion. Experimental Fermi surfaces were integrated within $\pm 10 \mathrm{meV}$ of the Fermi level. 
In addition to the bulk band structure, we have studied the surface states of $\mathrm{ZrP}_{2}$. Generally speaking, the appearance of surface states in ARPES depends on experimental conditions such as the quality and topography of the cleaved surface. This is of particular importance for three-dimensional materials like $\mathrm{ZrP}_{2}$, where cleaving may reveal different surface terminations [36]. Here, a small beam spot size can help to probe a single termination in the ARPES experiment. For this reason, we conducted additional ARPES studies using microARPES, the results of which are shown in Figs. 4(d)-4(f). The spectra are dominated by surface states, in contrast to the data shown in Figs. 4(a)-4(c). The projected bulk Fermi surface is enclosed by intense surface states, which disperse over more than $1 \mathrm{eV}$. Along $\overline{\mathrm{M}}-\overline{\mathrm{X}}-\overline{\mathrm{M}}$, the surface states are well separated from the projected bulk band structure, whereas they partly hybridize with the bulk along $\overline{\mathrm{Y}}-\bar{\Gamma}-\overline{\mathrm{Y}}$. Altogether, we find excellent agreement between the measured spectra and the slab calculations, considering a surface termination that exposes the phosphorus chains without breaking them [36].

The presence of both covalent and ionic bonding features in $\mathrm{ZrP}_{2}$, namely the phosphorus chains along the $b$ axis, indicates a significant degree of charge transfer from zirconium to phosphorus. However, in contrast to the insulating behavior favored by complete charge transfer, our transport and spectroscopic measurements clearly evidence a charge-compensated semimetallic ground state in $\mathrm{ZrP}_{2}$. The e-h compensation at low temperatures is further confirmed by band structure calculations and accounts for the unsaturated $\mathrm{XMR}$ [34]. In this regard, $\mathrm{ZrP}_{2}$ resembles the behavior of other early TMDPs such as NbAs $2 ~[2,5]$ and $\mathrm{WP}_{2}[1,43,44]$. With the finding of $\mathrm{XMR}$ in $\mathrm{ZrP}_{2}$, we are able to establish $\mathrm{XMR}$ as a general feature in early TMDPs. The underlying reason for this ubiquity is the combination of nonsymmorphic symmetry and incomplete charge transfer.

Strictly speaking, semimetallicity alone is not sufficient to explain the unsaturated XMR in all of the reported materials. Within the semiclassical two-band model, unsaturated MR is only possible if the e-h densities are exactly compensated [34]. In the more realistic cases, XMR is associated with the combination of moderate to perfect e-h compensation and sufficiently high carrier mobility. Such conditions are commonly fulfilled in topological semimetals, where the band crossings close to the Fermi energy give rise to very high carrier mobilities [Fig. 1(e)]. It has also been shown in several cases that an imbalanced e-h compensation can be offset by the very high carrier mobility, such that XMR is still observed [45-47]. Many of the early TMDPs were predicted and, in some cases, verified to host topologically nontrivial phases $[1,25,27,43,48]$. However, the coexistence of e-h compensation and nontrivial topology naturally complicates the disentanglement of their influence on transport, which has caused difficulties interpreting the origin of the XMR [29]. Based on our DFT calculations and ARPES measurements, we argue that $\mathrm{ZrP}_{2}$ is a clean case where the unsaturated XMR can be fully accounted for by trivial e-h compensation. Contributions from the nontrivial nodal loop can be excluded as it lies $\sim 70-230 \mathrm{meV}$ below the Fermi level. Additionally, open-orbit Fermi surface topology as in $\mathrm{MoAs}_{2}[3,49]$ can be ruled out as the origin. A similar situation was found for (a)

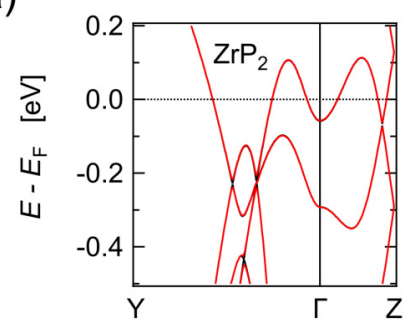

(c)

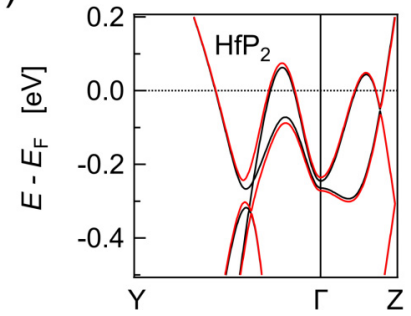

(b)

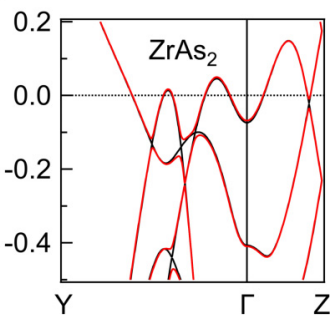

(d)

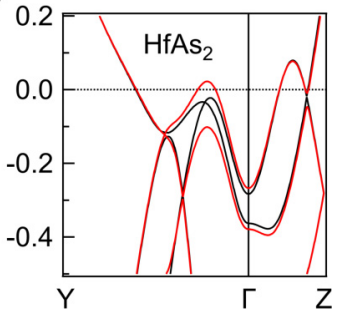

FIG. 5. Gap opening along the nodal loop through increased spin-orbit coupling (SOC). Band structures along $\mathrm{Y}-\Gamma-\mathrm{Z}$ for (a) $\mathrm{ZrP}_{2}$, (b) $\mathrm{ZrAs}_{2}$, (c) $\mathrm{HfP}_{2}$, and (d) $\mathrm{HfAs}_{2}$. Black lines: without SOC, red lines: with SOC. Experimentally determined crystal structures were used for calculations on $\mathrm{ZrP}_{2}$ [40] and $\mathrm{ZrAs}_{2}$ [51]. For $\mathrm{HfP}_{2}$ and $\mathrm{HfAs}_{2}$, the crystal structures of $\mathrm{ZrP}_{2}$ and $\mathrm{ZrAs}_{2}$ were used, respectively.

the type-II Weyl semimetal $\mathrm{WP}_{2}$ for which the XMR was attributed to e-h compensation despite the existence of Weyl nodes [43].

The direct observation of the nodal loop in the $k_{x}=0$ plane by ARPES was prevented by experimental limitations. Nevertheless, the excellent agreement between our calculations and the ARPES spectra provides a strong support for its presence in the low energy band structure of $\mathrm{ZrP}_{2}$. Inclusion of soft $\mathrm{x}$-ray ARPES in future investigations might be a powerful tool to reduce the $k_{z}$ broadening and resolve the nodal loop along $k_{z}$.

One might wonder about the topological nature of the surface states seen by ARPES and DFT. Nodal line semimetals are expected to host the so-called drumhead topological surface states [15]. However, the presence of a drumhead surface state is only guaranteed on the (100) surface of $\mathrm{ZrP}_{2}$, whereas $\mathrm{ZrP}_{2}$ naturally cleaves on the (001) surface. Together with the strongly termination-dependent connectivity of the (001) surface states found in our calculations, we conclude that the observed surface states are trivial in nature. This contrasts a recent report on $\mathrm{HfP}_{2}$ [50], a heavier homologue of $\mathrm{ZrP}_{2}$, where similar (001) surface states were interpreted as topological. Minor differences in the bulk band structures of $\mathrm{ZrP}_{2}$ and $\mathrm{HfP}_{2}$ may account for this contrast.

The structural similarity between $\mathrm{ZrP}_{2}$ and $\mathrm{HfP}_{2}$ can be further exploited. $\mathrm{ZrP}_{2}$ has three heavier homologues: $\mathrm{ZrAs}_{2}$, $\mathrm{HfP}_{2}$, and $\mathrm{HfAs}_{2}$ belonging to the same structure type [33,52]. The band structures are therefore very similar to $\mathrm{ZrP}_{2}$. In particular, all these materials feature a nodal loop in the $k_{x}=0$ plane (see Fig. 5). The strength of SOC, however, increases significantly between $\mathrm{ZrP}_{2}$ (lightest) and $\mathrm{HfAs}_{2}$ (heaviest). Accordingly, the gap opened along the nodal loop by SOC 
increases from $20 \mathrm{meV}$ in $\mathrm{ZrP}_{2}$ to $120 \mathrm{meV}$ in $\mathrm{HfAs}_{2}$. The $\mathrm{ZrP}_{2}$ family of materials thus provides a good platform to study the evolution of nodal loops with increasing SOC, an aspect of nodal loops that has received little attention so far. Furthermore, substitution of the pnictide with $\mathrm{Ge}, \mathrm{Si}$, and $\mathrm{Se}$ has been demonstrated [51,53,54], which opens the possibility of shifting the chemical potential by doping and access the topological crossings. We note that the corresponding diantimonides $\mathrm{ZrSb}_{2}$ and $\mathrm{HfSb}_{2}$ exist as well but have been shown to exhibit rather metallic behavior [55], presumably due to a lesser degree of charge transfer from zirconium or hafnium to antimony. In addition, the diantimonides crystallize in a different structure and space group than the diphosphides and diarsenides [56]. Hence, we cannot directly compare their band structures.

\section{CONCLUSION}

In summary, we demonstrated that $\mathrm{ZrP}_{2}$ is an e-h compensated nodal loop semimetal. By applying combined experimental and theoretical methods, we derived a consistent picture of the electronic structure and the resulting properties. The extremely large and unsaturated MR of $40000 \%$ at $2 \mathrm{~K}$ and $9 \mathrm{~T}$ is explained by trivial e-h compensation. Our band structure calculations, showing a nodal loop in the $k_{x}=0$ plane, are confirmed by ARPES experiments. However, contributions from nontrivial topology can be excluded as the origin of the XMR. Our findings establish unsaturated XMR as a general consequence of nonsymmorphic symmetry and incomplete charge transfer in early TMDPs. The group IV TMDPs, including $\mathrm{ZrP}_{2}$, stand out of this class of materials because of their tunability both in terms of strength of SOC and possibility to shift the chemical potential by doping. They thus provide an ideal platform for future investigations of nodal lines.

\section{ACKNOWLEDGMENTS}

We thank R. Koban and W. Schnelle for assistance with the magnetotransport measurements. This research was undertaken thanks in part to funding from the ERC Advanced Grant No. (742068) 'TOPMAT'; the Max Planck-UBC-UTokyo Centre for Quantum Materials and the Canada First Research Excellence Fund, Quantum Materials and Future Technologies Program, in addition to the Killam, Alfred P. Sloan, and Natural Sciences and Engineering Research Council of Canada's (NSERC's) Steacie Memorial Fellowships (A.D.); the Alexander von Humboldt Fellowship (A.D.); the Canada Research Chairs Program (A.D.); NSERC, Canada Foundation for Innovation (CFI); British Columbia Knowledge Development Fund (BCKDF); and the CIFAR Quantum Materials Program. This research also used resources of the Advanced Light Source, a U.S. DOE Office of Science User Facility under Contract No. DE-AC02-05CH11231.
[1] N. Kumar, Y. Sun, N. Xu, K. Manna, M. Yao, V. Suss, I. Leermakers, O. Young, T. Forster, M. Schmidt, H. Borrmann, B. Yan, U. Zeitler, M. Shi, C. Felser, and C. Shekhar, Extremely high magnetoresistance and conductivity in the type-II Weyl semimetals $\mathrm{WP}_{2}$ and $\mathrm{MoP}_{2}$, Nat. Commun. 8, 1642 (2017).

[2] Y.-Y. Wang, Q.-H. Yu, P.-J. Guo, K. Liu, and T.-L. Xia, Resistivity plateau and extremely large magnetoresistance in $\mathrm{NbAs}_{2}$ and $\mathrm{TaAs}_{2}$, Phys. Rev. B 94, 041103 (2016).

[3] R. Lou, Y. F. Xu, L.-X. Zhao, Z.-Q. Han, P.-J. Guo, M. Li, J.-C. Wang, B.-B. Fu, Z.-H. Liu, Y.-B. Huang, P. Richard, T. Qian, K. Liu, G.-F. Chen, H. M. Weng, H. Ding, and S.-C. Wang, Observation of open-orbit Fermi surface topology in the extremely large magnetoresistance semimetal $\mathrm{MoAs}_{2}$, Phys. Rev. B 96, 241106 (2017).

[4] J. Xu, N. J. Ghimire, J. S. Jiang, Z. L. Xiao, A. S. Botana, Y. L. Wang, Y. Hao, J. E. Pearson, and W. K. Kwok, Origin of the extremely large magnetoresistance in the semimetal YSb, Phys. Rev. B 96, 075159 (2017).

[5] K. Yokoi, H. Murakawa, M. Komada, T. Kida, M. Hagiwara, H. Sakai, and N. Hanasaki, Enhanced magnetoresistance in the binary semimetal $\mathrm{NbAs}_{2}$ due to improved crystal quality, Phys. Rev. Materials 2, 024203 (2018).

[6] Y. Luo, H. Li, Y. M. Dai, H. Miao, Y. G. Shi, H. Ding, A. J. Taylor, D. A. Yarotski, R. P. Prasankumar, and J. D. Thompson, Hall effect in the extremely large magnetoresistance semimetal $\mathrm{WTe}_{2}$, Appl. Phys. Lett. 107, 182411 (2015).

[7] H. Li, H.-W. Wang, H. He, J. Wang, and S.-Q. Shen, Giant anisotropic magnetoresistance and planar Hall effect in the Dirac semimetal $\mathrm{Cd}_{3} \mathrm{As}_{2}$, Phys. Rev. B 97, 201110 (2018).

[8] P. Li, C. H. Zhang, J. W. Zhang, Y. Wen, and X. X. Zhang, Giant planar Hall effect in the Dirac semimetal $\mathrm{ZrTe}_{5-\delta}$, Phys. Rev. B 98, 121108 (2018).

[9] N. P. Armitage, E. J. Mele, and A. Vishwanath, Weyl and Dirac semimetals in three-dimensional solids, Rev. Mod. Phys. 90, 015001 (2018).

[10] J. Hu, S.-Y. Xu, N. Ni, and Z. Mao, Transport of topological semimetals, Annu. Rev. Mater. Res. 49, 207 (2019).

[11] Z. K. Liu, J. Jiang, B. Zhou, Z. J. Wang, Y. Zhang, H. M. Weng, D. Prabhakaran, S. K. Mo, H. Peng, P. Dudin, T. Kim, M. Hoesch, Z. Fang, X. Dai, Z. X. Shen, D. L. Feng, Z. Hussain, and Y. L. Chen, A stable three-dimensional topological Dirac semimetal $\mathrm{Cd}_{3} \mathrm{As}_{2}$, Nat. Mater. 13, 677 (2014).

[12] Z. K. Liu, B. Zhou, Y. Zhang, Z. J. Wang, H. M. Weng, D. Prabhakaran, S. K. Mo, Z. X. Shen, Z. Fang, X. Dai, Z. Hussain, and Y. L. Chen, Discovery of a three-dimensional topological Dirac semimetal, $\mathrm{Na}_{3} \mathrm{Bi}$, Science 343, 864 (2014).

[13] S.-Y. Xu et al., Discovery of a Weyl fermion state with Fermi arcs in niobium arsenide, Nat. Phys. 11, 748 (2015).

[14] L. X. Yang, Z. K. Liu, Y. Sun, H. Peng, H. F. Yang, T. Zhang, B. Zhou, Y. Zhang, Y. F. Guo, M. Rahn, D. Prabhakaran, Z. Hussain, S. K. Mo, C. Felser, B. Yan, and Y. L. Chen, Weyl semimetal phase in the non-centrosymmetric compound TaAs, Nat. Phys. 11, 728 (2015).

[15] A. A. Burkov, M. D. Hook, and L. Balents, Topological nodal semimetals, Phys. Rev. B 84, 235126 (2011). 
[16] T. Bzdusek, Q. Wu, A. Ruegg, M. Sigrist, and A. A. Soluyanov, Nodal-chain metals, Nature 538, 75 (2016).

[17] Z. Yan, R. Bi, H. Shen, L. Lu, S.-C. Zhang, and Z. Wang, Nodallink semimetals, Phys. Rev. B 96, 041103 (2017).

[18] G. Chang, S.-Y. Xu, X. Zhou, S.-M. Huang, B. Singh, B. Wang, I. Belopolski, J. Yin, S. Zhang, A. Bansil, H. Lin, and M. Z. Hasan, Topological Hopf and Chain Link Semimetal States and Their Application to $\mathrm{Co}_{2} \mathrm{MnGa}$, Phys. Rev. Lett. 119, 156401 (2017).

[19] R. Bi, Z. Yan, L. Lu, and Z. Wang, Nodal-knot semimetals, Phys. Rev. B 96, 201305 (2017).

[20] T. Nakamura, S. Souma, Z. Wang, K. Yamauchi, D. Takane, H. Oinuma, K. Nakayama, K. Horiba, H. Kumigashira, T. Oguchi, T. Takahashi, Y. Ando, and T. Sato, Evidence for bulk nodal loops and universality of Dirac-node arc surface states in $\mathrm{ZrGe}_{c}\left(X_{c}=\mathrm{S}, \mathrm{Se}, \mathrm{Te}\right)$, Phys. Rev. B 99, 245105 (2019).

[21] L. M. Schoop, M. N. Ali, C. Strasser, A. Topp, A. Varykhalov, D. Marchenko, V. Duppel, S. S. Parkin, B. V. Lotsch, and C. R. Ast, Dirac cone protected by non-symmorphic symmetry and three-dimensional Dirac line node in $\mathrm{ZrSiS}$, Nat. Commun. 7, 11696 (2016).

[22] A. Topp, J. M. Lippmann, A. Varykhalov, V. Duppel, B. V. Lotsch, C. R. Ast, and L. M. Schoop, Non-symmorphic band degeneracy at the Fermi level in ZrSiTe, New J. Phys. 18, 125014 (2016).

[23] D. Takane, Z. Wang, S. Souma, K. Nakayama, C. X. Trang, T. Sato, T. Takahashi, and Y. Ando, Dirac-node arc in the topological line-node semimetal HfSiS, Phys. Rev. B 94, 121108 (2016).

[24] Y. Shao, Z. Sun, Y. Wang, C. Xu, R. Sankar, A. J. Breindel, C. Cao, M. M. Fogler, A. J. Millis, F. Chou, Z. Li, T. Timusk, M. B. Maple, and D. N. Basov, Optical signatures of Dirac nodal lines in $\mathrm{NbAs}_{2}$, Proc. Natl. Acad. Sci. USA 116, 1168 (2019).

[25] C. Xu, J. Chen, G.-X. Zhi, Y. Li, J. Dai, and C. Cao, Electronic structures of transition metal dipnictides $X \mathrm{Pn}_{2}(X=\mathrm{Ta}, \mathrm{Nb}$; $P n=$ P, As, Sb), Phys. Rev. B 93, 195106 (2016).

[26] J. Chen, Y.-K. Li, J. Dai, and C. Cao, Electronic structure and topological properties of centrosymmetric $\mathrm{MoAs}_{2} / \mathrm{WAs}_{2}$ from first principles, Sci. Rep. 7, 10491 (2017).

[27] B. Wang, B. Singh, B. Ghosh, W.-C. Chiu, M. M. Hosen, Q. Zhang, L. Ying, M. Neupane, A. Agarwal, H. Lin, and A. Bansil, Topological crystalline insulator state with type-II Dirac fermions in transition metal dipnictides, Phys. Rev. B 100, 205118 (2019).

[28] Z. Yuan, H. Lu, Y. Liu, J. Wang, and S. Jia, Large magnetoresistance in compensated semimetals $\mathrm{TaAs}_{2}$ and $\mathrm{NbAs}_{2}$, Phys. Rev. B 93, 184405 (2016).

[29] K. Wang, D. Graf, L. Li, L. Wang, and C. Petrovic, Anisotropic giant magnetoresistance in $\mathrm{NbSb}_{2}$, Sci. Rep. 4, 7328 (2014).

[30] Y. Li, L. Li, J. Wang, T. Wang, X. Xu, C. Xi, C. Cao, and J. Dai, Resistivity plateau and negative magnetoresistance in the topological semimetal TaSb 2 , Phys. Rev. B 94, 121115 (2016).

[31] J. Du, Z. Lou, S. N. Zhang, Y. Zhou, B. Xu, Q. Chen, Y. Tang, S. Chen, H. Chen, Q. Zhu, H. Wang, J. Yang, Q. S. Wu, O. V. Yazyev, and M. Fang, Extremely large magnetoresistance in the topologically trivial semimetal $\alpha-\mathrm{WP}_{2}$, Phys. Rev. B 97, 245101 (2018).

[32] F. Hulliger, New representatives of the $\mathrm{NbAs}_{2}$ and $\mathrm{ZrAs}_{2}$ structures, Nature 204, 775 (1964).
[33] F. Hulliger, Crystal chemistry of the chalcogenides and pnictides of the transition elements, in Structure and Bonding (Springer, Berlin, Heidelberg, 1968), pp. 83-229.

[34] A. Pippard, in Cambridge Studies in Low Temperature Physics: Magnetoresistance in Metals, edited by A. M. Goldman, P. V. E. McClintock, and M. Springford (Cambridge University Press, Cambridge, 1989).

[35] I. A. Leahy, Y.-P. Lin, P. E. Siegfried, A. C. Treglia, J. C. W. Song, R. M. Nandkishore, and M. Lee, Nonsaturating large magnetoresistance in semimetals, Proc. Natl. Acad. Sci. USA 115, 10570 (2018).

[36] See Supplemental Material at http://link.aps.org/supplemental/ 10.1103/PhysRevB.103.155144 for details of the crystal characterization, $k_{z}$ dependence of the ARPES data, and surface band structures for all possible terminations.

[37] P. Blaha, K. Schwarz, G. Madsen, D. Kvasnicka, J. Luitz, R. Laskowski, F. Tran, and L. Marks, WIEN2k, An Augmented Plane Wave + Local Orbitals Program for Calculating Crystal Properties (Technische Universität Wien, Austria, 2019).

[38] J. P. Perdew, K. Burke, and M. Ernzerhof, Generalized Gradient Approximation Made Simple, Phys. Rev. Lett. 77, 3865 (1996).

[39] F. Tran and P. Blaha, Accurate Band Gaps of Semiconductors and Insulators with a Semilocal Exchange-Correlation Potential, Phys. Rev. Lett. 102, 226401 (2009).

[40] M. Huber and H. J. Deiseroth, Crystal structure of zirconium diphosphide, $\mathrm{ZrP}_{2}$, Z. Kristallogr. 209, 370 (1994).

[41] V. N. Strocov, Intrinsic accuracy in 3-dimensional photoemission band mapping, J. Electron Spectrosc. Relat. Phenom. 130, 65 (2003).

[42] A. Damascelli, Probing the electronic structure of complex systems by ARPES, Phys. Scr. 2004, 61 (2004).

[43] E. Razzoli, B. Zwartsenberg, M. Michiardi, F. Boschini, R. P. Day, I. S. Elfimov, J. D. Denlinger, V. Süss, C. Felser, and A. Damascelli, Stable Weyl points, trivial surface states, and particle-hole compensation in $\mathrm{WP}_{2}$, Phys. Rev. B 97, 201103 (2018).

[44] R. Schönemann, N. Aryal, Q. Zhou, Y.-C. Chiu, K.-W. Chen, T. J. Martin, G. T. McCandless, J. Y. Chan, E. Manousakis, and L. Balicas, Fermi surface of the Weyl type-II metallic candidate $\mathrm{WP}_{2}$, Phys. Rev. B 96, 121108 (2017).

[45] J. Jiang, N. B. M. Schröter, S.-C. Wu, N. Kumar, C. Shekhar, H. Peng, X. Xu, C. Chen, H. F. Yang, C.-C. Hwang, S.-K. Mo, C. Felser, B. H. Yan, Z. K. Liu, L. X. Yang, and Y. L. Chen, Observation of topological surface states and strong electron/hole imbalance in extreme magnetoresistance compound LaBi, Phys. Rev. Materials 2, 024201 (2018).

[46] T. Liang, Q. Gibson, M. N. Ali, M. Liu, R. J. Cava, and N. P. Ong, Ultrahigh mobility and giant magnetoresistance in the Dirac semimetal $\mathrm{Cd}_{3} \mathrm{As}_{2}$, Nat. Mat. 14, 280 (2015).

[47] F. F. Tafti, Q. D. Gibson, S. K. Kushwaha, N. Haldolaarachchige, and R. J. Cava, Resistivity plateau and extreme magnetoresistance in LaSb, Nat. Phys. 12, 272 (2016).

[48] D. Gresch, Q. Wu, G. W. Winkler, and A. A. Soluyanov, Hidden Weyl points in centrosymmetric paramagnetic metals, New J. Phys. 19, 035001 (2017).

[49] R. Singha, A. Pariari, G. K. Gupta, T. Das, and P. Mandal, Probing the Fermi surface and magnetotransport properties of $\mathrm{MoAs}_{2}$, Phys. Rev. B 97, 155120 (2018). 
[50] C. Sims, M. M. Hosen, H. Aramberri, C.-Y. Huang, G. Dhakal, K. Dimitri, F. Kabir, S. Regmi, X. Zhou, T.-R. Chang, H. Lin, D. Kaczorowski, N. Kioussis, and M. Neupane, Terminationdependent topological surface states in nodal-loop semimetal HfP $_{2}$, Phys. Rev. Materials 4, 054201 (2020).

[51] P. E. R. Blanchard, R. G. Cavell, and A. Mar, On the existence of $\mathrm{ZrAs}_{2}$ and ternary extension $\mathrm{Zr}\left(\mathrm{Ge}_{x} \mathrm{As}_{1-x}\right) \mathrm{As}(0<x<0.4)$, J. Alloys Compd. 505, 17 (2010).

[52] W. Jeitschko and H. Nowotny, Die Kristallarten HfAs und HfAs $_{2}$, Monatsh. Chem. 93, 1284 (1962).

[53] M. W. Gaultois, A. P. Grosvenor, P. E. R. Blanchard, and A. Mar, Ternary arsenides $\mathrm{Zr}\left(\mathrm{Si}_{x} \mathrm{As}_{1-x}\right) \mathrm{As}$ with $\mathrm{PbCl}_{2}$-type $(0<x<0.4)$ and PbFCl-type $(x=0.6)$ structures, J. Alloys Compd. 492, 19 (2010).

[54] S. Ishida, H. Fujihisa, I. Hase, Y. Yanagi, K. Kawashima, K. Oka, Y. Gotoh, Y. Yoshida, A. Iyo, H. Eisaki, and H. Kito, Superconductivity in layered $\mathrm{ZrP}_{2-x} \mathrm{Se}_{x}$ with PbFCl-type structure, Supercond. Sci. Technol. 29, 055004 (2016).

[55] L.-L. Sun, Y.-Y. Wang, S. Xu, and T.-L. Xia, Crystal growth and magneto-transport properties of $\alpha-\mathrm{ZrSb}_{2}$ and $\alpha-\mathrm{HfSb}_{2}$, Europhys. Lett. 120, 37002 (2017).

[56] A. Kjekshus, On the Crystal structures of $\mathrm{ZrSb}_{2}$ and $\alpha-\mathrm{HfSb}_{2}$, Acta Chem. Scand. 26, 1633 (1972). 